\title{
The Frontal Assessment Battery (FAB) and its sub-scales: validation and updated normative data in an Italian population sample
}

\author{
Edoardo Nicolò Aiello ${ }^{1,2}$ ( $\cdot$ Antonella Esposito $^{3}$. Chiara Gramegna ${ }^{3} \cdot$ Valentina Gazzaniga $^{3}$ - Stefano Zago ${ }^{4,5}$. \\ Teresa Difonzo ${ }^{4}$.lldebrando Marco Appollonio ${ }^{1,5,6} \cdot$ Nadia Bolognini $^{3,5,7}$ (I)
}

Received: 21 March 2021 / Accepted: 7 June 2021 / Published online: 29 June 2021

(c) The Author(s) 2021

\begin{abstract}
Background Deficits of executive functioning (EF) are frequently found in neurological disorders. The Frontal Assessment Battery $(\mathrm{FAB})$ is one of the most widespread and psychometrically robust EF screeners in clinical settings. However, in Italy, FAB norms date back to 15 years ago; moreover, its validity against "EF-loaded" global cognitive screeners (e.g., the Montreal Cognitive Assessment, MoCA) has yet to be tested. This study thus aimed at (a) providing updated normative data for the Italian FAB and (b) assessing its convergent validity with the MoCA.

Methods Four-hundred and seventy-five healthy Italian native speakers (306 females, 169 males; mean age: $61.08 \pm 15.1$; mean education: $11.67 \pm 4.57$ ) were administered by the MoCA and the FAB. FAB items were divided into three subscales: FAB-1 (linguistically mediated EF), FAB-2 (planning), and FAB-3 (inhibition). Regression-based norms were derived (equivalent scores) for all $\mathrm{FAB}$ measures.

Results Age and education were predictive of all FAB measures, whereas no gender differences were detected. The FAB and its sub-scales were related to MoCA measures - the strongest associations being found with MoCA total and MoCA-EF scores. FAB sub-scales were both internally related and associated with FAB total scores.

Discussion The FAB proved to have convergent validity with both global cognitive and EF measures in healthy individuals. The present study provides updated normative data for the FAB and its sub-scales in an Italian population sample, and thus supports an adaptive usage of this EF screener.
\end{abstract}

Keywords Frontal Assessment Battery · Frontal lobes $\cdot$ Executive functioning $\cdot$ Normative data $\cdot$ Dysexecutive symptoms . Neuropsychological assessment

Edoardo Nicolò Aiello and Antonella Esposito contributed equally to this work

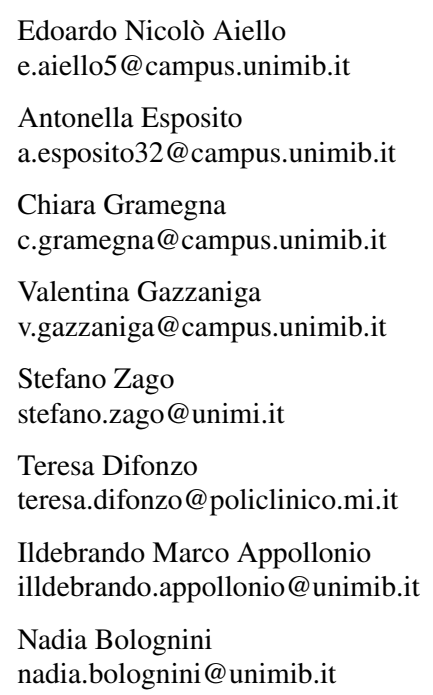

1 School of Medicine and Surgery, University of MilanoBicocca, Monza, Italy

2 Clinical Neuroscience, University of Milano-Bicocca, Monza, Italy

3 Department of Psychology, University of Milano-Bicocca, Milan, Italy

4 Fondazione IRCCS Ca' Granda Ospedale Maggiore Policlinico, University of Milan, Milan, Italy

5 Milan Center for Neuroscience (NeuroMI), Milan, Italy

6 Neurology Section, School of Medicine and Surgery, University of Milano-Bicocca, Monza, Italy

7 Neuropsychological Laboratory, IRCCS Istituto Auxologico Italiano, Milan, Italy 


\section{Introduction}

Executive functioning (EF) comprises a multifaceted set of frontally mediated, noninstrumental cognitive processes that control instrumental domains and behavior [1]. Executive disorders are thus frequently found in a variety of neurological conditions of different etiologies that affect cortical/ subcortical frontal structures [2].

Although second-level specific psychometric tests are to be preferred when assessing EF [3], screening instruments for executive deficits are often useful in clinical settings, such as providing with an optimal trade-off between informativity and both sensitivity and a rapid administration [4].

The Frontal Assessment Battery (FAB) [5] is an EF screener that requires 5-10' to administer and consists of 6 tasks assessing different EF facets: (1) concept formation and abstract reasoning (similarities); (2) mental flexibility (phonemic verbal fluency); (3) motor programming (Luria motor sequences); (4) sensitivity to interference (conflicting instructions); (5) inhibitory control (go-no-go); (6) environmental autonomy (prehension behavior) $[5,6]$.

The FAB is one of the most widely used EF screeners worldwide; its psychometric properties, clinical usability, and neural correlates have been thoroughly investigated [7].

In Italy, the FAB has been adapted and normed, as well as validated in both healthy and clinical populations $[6,8]$. However, current FAB Italian normative data date back to more than 15 years ago and sociodemographic changes require norms updating [9]. Moreover, to the best of the authors' knowledge, the FAB has been only validated against "non-executive" screeners in Italy [6]-e.g., the Mini-Mental State Examination (MMSE) [10]—whereas its association with "EF-loaded" screening measures, e.g., the Montreal Cognitive Assessment (MoCA) [11], has yet to be explored. In addition, FAB normative studies do not provide with norms for its subtests, despite this being an increasingly widespread approach for cognitive screeners in Italy [12, 13], as it allows greater flexibility for clinicians when using these instruments.
Accordingly, the two aims of the present study are (1) providing updated normative data for both FAB total and sub-test scales in a large Italian representative population sample and (2) validating the FAB and its subscales against the MoCA.

\section{Subjects and methods}

\section{Participants}

The sample consisted of $N=475$ healthy Italian native speakers from different provinces of Northern Italy (Table 1). Participants had no history of neurological, psychiatric, or severe general medical conditions (i.e. severe internal and metabolic morbidities or systemic/organ failures). Studies that data come from were approved by the Research Evaluation Committee of the Department of Psychology of University of Milano-Bicocca on behalf of the Ethical Committee of the same university. Participants provided informed consent to participation and signed a data treatment disclaimer research purposes.

\section{Materials}

Global cognition was assessed via the MoCA [12, 14], which encompasses subtests evaluating EF (MoCA-EF), attention (MoCA-A), language (MoCA-L), memory (MoCA-M), visuospatial functions (MoCA-VS), and orientation (MoCA-O). Supplementary Table 1 provides the protocol for the current FAB. FAB items $(N=6)$ were grouped into 3 subscales: FAB-1 comprising the first two items (similarities and phonemic verbal fluency, linguistically mediated EF); FAB-2, comprising the second two-item set (Luria motor sequences and conflicting instructions, planning); FAB-3, comprising the last two items (go-no-go and prehension behavior, inhibition). All participants were administered the MoCA first and then the FAB.

Table 1 Sample stratification for age, education, and $\operatorname{sex}(\mathrm{M} / \mathrm{F}: \mathrm{M}=$ males, $\mathrm{F}=$ females)

\begin{tabular}{|c|c|c|c|c|c|c|c|c|}
\hline \multirow[b]{2}{*}{ Education } & \multicolumn{8}{|c|}{ Age $(\mathrm{M} / \mathrm{F})$} \\
\hline & $35 \leq$ & $36-45$ & $46-55$ & $56-65$ & $66-75$ & $76-85$ & $86-95$ & $>95$ \\
\hline $5 \leq$ & $0 / 0$ & $0 / 1$ & $0 / 0$ & $0 / 3$ & $1 / 18$ & $11 / 33$ & $4 / 5$ & $1 / 0$ \\
\hline $6-11$ & $1 / 1$ & $6 / 5$ & $9 / 26$ & $20 / 15$ & $5 / 10$ & $6 / 21$ & $4 / 2$ & $0 / 0$ \\
\hline $12-16$ & $6 / 7$ & $4 / 8$ & $20 / 42$ & $31 / 33$ & $7 / 5$ & $5 / 9$ & $1 / 6$ & $0 / 0$ \\
\hline $17-21$ & $3 / 5$ & $1 / 7$ & $6 / 13$ & $17 / 22$ & $0 / 1$ & $1 / 2$ & $0 / 2$ & $0 / 0$ \\
\hline$>21$ & $0 / 0$ & $0 / 2$ & $0 / 2$ & $0 / 0$ & $0 / 0$ & $0 / 0$ & $0 / 0$ & $0 / 0$ \\
\hline
\end{tabular}

Cells show male/female ratio for each co-occurrence 
Table 2 Participants' background and cognitive scores

\begin{tabular}{|c|c|c|c|c|c|c|c|c|}
\hline$N$ & $\operatorname{Sex}(\mathrm{F} / \mathrm{M})$ & Age (years) & Education (years) & MoCA & FAB & FAB-1 & FAB-2 & FAB-3 \\
\hline 475 & $306 / 169$ & $\begin{array}{c}61.08 \pm 15.1 \\
(21-96)\end{array}$ & $\begin{array}{c}11.67 \pm 4.57 \\
(1-25)\end{array}$ & $\begin{array}{c}24.5 \pm 3.95 \\
\quad(8-30)\end{array}$ & $\begin{array}{c}15.9 \pm 2.17 \\
(9-18)\end{array}$ & $\begin{array}{c}5.21 \pm 0.93 \\
(1-6)\end{array}$ & $\begin{array}{c}5.41 \pm 1.08 \\
(0-6)\end{array}$ & $\begin{array}{c}5.27 \pm 1.14 \\
(1-6)\end{array}$ \\
\hline
\end{tabular}

$F$ female; $M$ male; $M o C A$ Montreal Cognitive Assessment; FAB Frontal Assessment Battery; FAB-1, comprises the first two items (similarities and phonemic verbal fluency); FAB-2, the second two-item set (Luria motor sequences and conflicting instructions); FAB-3, the last two items (go-no-go and prehension behavior)

\section{Statistical analyses}

According to previous normative studies $[15,16]$, the minimum sample size was set at $N=287$ by means of a power analysis $\left(\alpha=0.05 ; 1-\beta=0.9 ; f^{2}=0.05\right)$ for multiple linear regression $\left(d f_{\text {numerator }}=3\right)$ analyses [17] via the $\mathrm{R}$ 3.6.3 package $p w r[18]$.

Normality assumptions on both background and cognitive raw variables were checked by evaluating skewness and kurtosis values (judged as abnormal if $\geq|1|$ and $|3|$, respectively) [19].

Associations of interest between quantitative variables were assessed by means of either Pearson's or Spearman's techniques. When judged as relevant, Bonferroni correction for multiple comparisons was applied.

According to the equivalent scores (ES) method [20, 21], raw scores (RSs) were adjusted for significant intervening background predictors (or their transforms) via regression-based equations. The cutoff was identified by computing outer and inner tolerance limits (oTL; iTL). Adjusted scores (ASs) were then standardized into a 5-level quasi-continuous scale: $\mathrm{ES}=0$ (ASs $\leq \mathrm{oTL}$; "abnormal"); $\mathrm{ES}=4$ (ASs $>M d n$; "normal"); $\mathrm{ES}=1,2$, and 3 (oTL $<$ ASs $\leq M d n$; respectively, "borderline," "low-end normal," "normal").

Analyses were performed via SPSS 27 [22] and R 3.6.3 [23]. Regression studies, as well as computations of both
TLs and ES thresholds, were implemented according to guidelines and software solutions described in [24].

\section{Results}

Background and cognitive scores are summarized in Table 2. The vast majority of FAB scales were related to MoCA measures (Table 3): the strongest associations were found with MoCA-EF and total scores. FAB subtest scores were all associated with each other $\left(0.21 \leq r_{s}(475) \leq 0.24 ; p<0.001\right)$, as well as with FAB total scores $\left(\mathrm{FAB}-1: r_{s}(475)=0.65\right.$; FAB-2: $r_{s}(475)=0.63$; FAB-3: $\left.r_{s}(475)=0.7\right)$.

Age was negatively related to FAB-1 $\left(r_{s}(475)=-0.3\right.$; $p<0.001),-2 \quad\left(r_{s}(475)=-0.36 ; p<0.001\right),-3$ $\left(r_{s}(475)=-0.3 ; p<0.001\right)$ and total $\left(r_{s}(475)=-0.44\right.$; $p<0.001)$ scores, whereas a positive association with education was detected: FAB- $1\left(r_{s}(475)=0.35 ; p<0.001\right)$, FAB-2 $\left(r_{s}(475)=0.24 ; p<0.001\right)$, FAB $-3\left(r_{s}(475)=0.31\right.$; $p<0.001)$, and total $\left(r_{s}(475)=0.42 ; p<0.001\right)$. No sex differences were found: FAB- $1(t(473)=-0.92 ; p=0.357)$, FAB-2 $(t(473)=0.44 ; p=0.66)$, FAB-3 $(t(385.3)=1.5$; $p=0.25)$, and total $(t(473)=0.4 ; p=0.689)$.

When simultaneously tested, age and education revealed to be predictive of FAB both total and subtest scores (age: $-0.17 \leq \beta \leq-0.34 ; p \leq 0.001$; education: $|0.15| \leq \beta \leq|0.33| ; p \leq 0.001)$. Cubic age and logarithmic
Table 3 Correlations between FAB and MoCA scores

\begin{tabular}{llccccccl}
\hline & & MoCA & MoCA-EF & MoCA-L & MoCA-A & MoCA-M & MoCA-VS & MoCA-O \\
\hline FAB & $r_{s}$ & 0.49 & 0.46 & 0.35 & 0.32 & 0.32 & 0.28 & 0.19 \\
& $p$ & $<0.001$ & $<0.001$ & $<0.001$ & $<0.001$ & $<0.001$ & $<0.001$ & $<0.001$ \\
FAB-1 & $r_{s}$ & 0.4 & 0.48 & 0.24 & 0.2 & 0.23 & 0.28 & 0.12 \\
& $p$ & $<0.001$ & $<0.001$ & $<0.001$ & $<0.001$ & $<0.001$ & $<0.001$ & n.s \\
FAB-2 & $r_{s}$ & 0.35 & 0.28 & 0.27 & 0.27 & 0.23 & 0.18 & 0.18 \\
& $p$ & $<0.001$ & $<0.001$ & $<0.001$ & $<0.001$ & $<0.001$ & $<0.001$ & $<0.001$ \\
FAB-3 & $r_{s}$ & 0.3 & 0.27 & 0.22 & 0.22 & 0.21 & 0.18 & 0.11 \\
& $p$ & $<0.001$ & $<0.001$ & $<0.001$ & $<0.001$ & $<0.001$ & $<0.001$ & n.s \\
\hline
\end{tabular}

$F A B$ Frontal Assessment Battery (numbers following the acronym represent subtests); MoCA Montreal Cognitive Assessment; $E F$ executive functioning; $L$ language; $A$ attention; $M$ memory; $O$ orientation; $V S$ visuospatial. $\alpha_{\text {adjusted }}$ was set at $0.0017(\mathrm{a} / k=0.05 / 28) ; n . s$. not significant at $\alpha_{\text {adjusted }}$ 
Table 4 Adjustment grid according to age and education for FAB total raw score

\begin{tabular}{|c|c|c|c|c|c|c|c|c|c|c|c|c|c|}
\hline \multirow[t]{2}{*}{ Education } & \multicolumn{13}{|l|}{ Age } \\
\hline & 35 & 40 & 45 & 50 & 55 & 60 & 65 & 70 & 75 & 80 & 85 & 90 & 95 \\
\hline 5 & 0.28 & 0.36 & 0.47 & 0.61 & 0.77 & 0.97 & 1.21 & 1.48 & 1.79 & 2.15 & 2.56 & 3.02 & 3.54 \\
\hline 8 & -0.46 & -0.37 & -0.26 & -0.13 & 0.04 & 0.23 & 0.47 & 0.74 & 1.06 & 1.42 & 1.83 & 2.29 & 2.8 \\
\hline 11 & -0.96 & -0.87 & -0.76 & -0.63 & -0.46 & -0.26 & -0.03 & 0.24 & 0.56 & 0.92 & 1.33 & 1.79 & 2.3 \\
\hline 13 & -1.22 & -1.13 & -1.02 & -0.89 & -0.72 & -0.53 & -0.29 & -0.02 & 0.3 & 0.66 & 1.07 & 1.53 & 2.04 \\
\hline 16 & -1.54 & -1.46 & -1.35 & -1.21 & -1.05 & -0.85 & -0.62 & -0.34 & -0.03 & 0.33 & 0.74 & 1.2 & 1.71 \\
\hline 18 & -1.73 & -1.64 & -1.53 & -1.4 & -1.23 & -1.03 & -0.8 & -0.53 & -0.21 & 0.15 & 0.56 & 1.02 & 1.53 \\
\hline 21 & -1.97 & -1.88 & -1.78 & -1.64 & -1.47 & -1.28 & -1.04 & -0.77 & -0.45 & -0.09 & 0.32 & 0.78 & 1.29 \\
\hline
\end{tabular}

$F A B$ Frontal Assessment Battery. Adjusted score=raw score $+0.000004 *\left[\left(a g e^{\wedge} 3\right)-269,630.547368\right]-1.565729 *[\ln ($ education$)-2.366383]$. Significant decimals of adjustment factors are displayed. Adjustment factors have been extracted from the aforementioned formula and do not always reflect empirical co-occurrences

education proved to be the most significant predictors of all FAB scales, with the exception of FAB-2 and FAB-3 that were best predicted by reciprocal education and quadratic age, respectively (Tables 4 and 5).
Selected correction factors and adjustment equations for FAB total and subtest RSs are displayed in Tables 4 and 5, respectively. TLs and ESs classifications for all FAB ASs are reported in Table 6.

Table 5 Adjustment grids according to age and education for FAB-1, FAB-2, and FAB-3 raw scores

\begin{tabular}{|c|c|c|c|c|c|c|c|c|c|c|c|c|c|c|}
\hline Subtest & & Age & & & & & & & & & & & & \\
\hline \multirow[t]{8}{*}{ FAB-1 } & Education & 35 & 40 & 45 & 50 & 55 & 60 & 65 & 70 & 75 & 80 & 85 & 90 & 95 \\
\hline & 5 & 0.23 & 0.25 & 0.28 & 0.32 & 0.36 & 0.41 & 0.46 & 0.53 & 0.61 & 0.7 & 0.8 & 0.92 & 1.05 \\
\hline & 8 & -0.05 & -0.03 & - & 0.03 & 0.07 & 0.12 & 0.18 & 0.25 & 0.33 & 0.42 & 0.52 & 0.63 & 0.76 \\
\hline & 11 & -0.25 & -0.22 & -0.2 & -0.16 & -0.12 & -0.07 & -0.01 & 0.05 & 0.13 & 0.22 & 0.33 & 0.44 & 0.57 \\
\hline & 13 & -0.35 & -0.33 & -0.3 & -0.27 & -0.22 & -0.17 & -0.12 & -0.05 & 0.03 & 0.12 & 0.22 & 0.34 & 0.47 \\
\hline & 16 & -0.47 & -0.45 & -0.43 & -0.39 & -0.35 & -0.3 & -0.24 & -0.17 & -0.09 & - & 0.1 & 0.21 & 0.34 \\
\hline & 18 & -0.54 & -0.52 & -0.5 & -0.46 & -0.42 & -0.37 & -0.31 & -0.24 & -0.17 & -0.08 & 0.03 & 0.14 & 0.27 \\
\hline & 21 & -0.64 & -0.62 & -0.59 & -0.56 & -0.52 & -0.47 & -0.41 & -0.34 & -0.26 & -0.17 & -0.07 & 0.05 & 0.18 \\
\hline \multirow[t]{7}{*}{ FAB-2 } & 5 & -0.21 & -0.17 & -0.12 & -0.05 & 0.03 & 0.13 & 0.25 & 0.39 & 0.54 & 0.72 & 0.93 & 1.16 & 1.41 \\
\hline & 8 & -0.4 & -0.36 & -0.31 & -0.24 & -0.16 & -0.06 & 0.06 & 0.2 & 0.35 & 0.53 & 0.74 & 0.97 & 1.23 \\
\hline & 11 & -0.49 & -0.45 & -0.39 & -0.33 & -0.24 & -0.14 & -0.03 & 0.11 & 0.27 & 0.45 & 0.65 & 0.88 & 1.14 \\
\hline & 13 & -0.53 & -0.48 & -0.43 & -0.36 & -0.28 & -0.18 & -0.06 & 0.07 & 0.23 & 0.41 & 0.62 & 0.85 & 1.1 \\
\hline & 16 & -0.56 & -0.52 & -0.47 & -0.4 & -0.31 & -0.22 & -0.1 & 0.04 & 0.2 & 0.38 & 0.58 & 0.81 & 1.07 \\
\hline & 18 & -0.58 & -0.54 & -0.48 & -0.42 & -0.33 & -0.23 & -0.12 & 0.02 & 0.18 & 0.36 & 0.56 & 0.79 & 1.05 \\
\hline & 21 & -0.6 & -0.56 & -0.5 & -0.44 & -0.35 & -0.25 & -0.14 & - & 0.16 & 0.34 & 0.54 & 0.77 & 1.03 \\
\hline \multirow[t]{7}{*}{ FAB-3 } & 5 & 0.2 & 0.24 & 0.29 & 0.33 & 0.39 & 0.45 & 0.51 & 0.58 & 0.66 & 0.74 & 0.82 & 0.91 & 1.01 \\
\hline & 8 & -0.1 & -0.06 & -0.02 & 0.03 & 0.09 & 0.15 & 0.21 & 0.28 & 0.36 & 0.44 & 0.52 & 0.61 & 0.71 \\
\hline & 11 & -0.3 & -0.26 & -0.22 & -0.17 & -0.12 & -0.06 & 0.01 & 0.08 & 0.15 & 0.23 & 0.32 & 0.41 & 0.5 \\
\hline & 13 & -0.41 & -0.37 & -0.33 & -0.28 & -0.22 & -0.16 & -0.1 & -0.03 & 0.04 & 0.12 & 0.21 & 0.3 & 0.39 \\
\hline & 16 & -0.54 & -0.5 & -0.46 & -0.41 & -0.36 & -0.3 & -0.23 & -0.16 & -0.09 & -0.01 & 0.08 & 0.17 & 0.26 \\
\hline & 18 & -0.62 & -0.58 & -0.53 & -0.49 & -0.43 & -0.37 & -0.31 & -0.24 & -0.16 & -0.08 & - & 0.1 & 0.19 \\
\hline & 21 & -0.72 & -0.68 & -0.63 & -0.58 & -0.53 & -0.47 & -0.41 & -0.34 & -0.26 & -0.18 & -0.1 & -0.01 & 0.09 \\
\hline
\end{tabular}

$F A B$ Frontal Assessment Battery; the number following the acronym indicates the subscale in exam. FAB-1 adjusted score $=$ raw score $+0.000001 *\left[\left(\right.\right.$ age $\left.\left.^{\wedge} 3\right)-269,630.547368\right]-0.607345^{*}[\ln ($ education $)-2.366383] . \quad$ FAB-2 adjusted score $=$ raw score $+0.000002 *\left[\left(\right.\right.$ age $\left.e^{\wedge} 3\right)-$ $269,630.547368]+2.527494 *[(1 /$ education $)-0.105356] . \quad$ FAB-3 $\quad$ adjusted $\quad$ score $=$ raw $\quad$ score $+0.000103 *\left[\left(\right.\right.$ age $\left.\left.{ }^{\wedge} 2\right)-3958.627368\right]-$ $0.640471 *[\ln$ (education)-2.366383]. Significant decimals of adjustment factors are displayed. Adjustment factors have been extracted from the aforementioned formula and do not always reflect empirical co-occurrences 
Table 6 Equivalent Scores for the FAB-T, FAB-1, FAB-2, and FAB-3 adjusted scores

\begin{tabular}{|c|c|c|c|c|c|c|c|}
\hline \multicolumn{8}{|c|}{ Equivalent scores } \\
\hline & oTL & iTL & 0 & 1 & 2 & 3 & 4 \\
\hline FAB & 12.02 & 13.16 & $\leq 12.02$ & $12.03-13.71$ & $13.72-15.1$ & $15.11-16.24$ & $\geq 16.25$ \\
\hline FAB-1 & 3.49 & 3.81 & $\leq 3.49$ & $3.5-4.35$ & $4.36-4.76$ & $4.77-5.49$ & $\geq 5.5$ \\
\hline FAB-2 & 2.74 & 3.76 & $\leq 2.74$ & $2.75-4.45$ & $4.46-5.41$ & $5.42-5.69$ & $\geq 5.7$ \\
\hline FAB-3 & 2.8 & 3.12 & $\leq 2.8$ & $2.81-3.57$ & $3.58-5.09$ & $5.1-5.65$ & $\geq 5.66$ \\
\hline
\end{tabular}

$F A B-T$ Frontal Assessment Battery (numbers following the acronym represent subtests); oTL outer tolerance limit; iTL inner tolerance limit

\section{Discussion}

The present work provides Italian practitioners with updated normative data for the FAB and its subtests. These norms cover a wider age and education range and are drawn from a larger sample size $(N=475)$ than those of previous normative studies $(N=236$ [8] and $N=364$ [6]). Moreover, norms for FAB subscales are provided - this representing a previously unreported feature that supports an adaptive usage of the screener.

This study overall replicates previous findings with respect to the range of FAB scores' predictors in the Italian population: the performance increases with higher educational attainment and decreases with aging, while no sex differences are detected[6, 8]. It has nonetheless to be noted that the cutoff reported here (12.03), despite being similar to that derived by Iavarone et al. [8] (11.54), is more conservative than Appollonio et al.'s [6] (13.5). This aspect might reflect sociodemographic changes that have occurred in the last two decades in northern Italian population.

To the best of the authors' knowledge, this contribution is the first showing convergent validity between the FAB (and its sub-scales) and an "EF-loaded" cognitive screeneri.e., the MoCA — in Italian healthy individuals. The present results also support the notion of the MoCA being a screening instrument sensitive to EF deficits, when compared to other screeners, such as the MMSE [25].

Supplementary Information The online version contains supplementary material available at https://doi.org/10.1007/s10072-021-05392-y.

Funding Open access funding provided by Università degli Studi di Milano - Bicocca within the CRUI-CARE Agreement.

Data Availability Data collected and analyzed during the present study are available on the Open Science Framework (OSF) repository (https://osf.io/4xypv/).

\section{Declarations}

Ethics approval This study was conducted in accordance with the Declaration of Helsinki. Participants provided their informed consent to participation.
Conflicts of interests The authors declare no competing interests.

Open Access This article is licensed under a Creative Commons Attribution 4.0 International License, which permits use, sharing, adaptation, distribution and reproduction in any medium or format, as long as you give appropriate credit to the original author(s) and the source, provide a link to the Creative Commons licence, and indicate if changes were made. The images or other third party material in this article are included in the article's Creative Commons licence, unless indicated otherwise in a credit line to the material. If material is not included in the article's Creative Commons licence and your intended use is not permitted by statutory regulation or exceeds the permitted use, you will need to obtain permission directly from the copyright holder. To view a copy of this licence, visit http://creativecommons.org/licenses/by/4.0/.

\section{References}

1. Jurado MB, Rosselli M (2007) The elusive nature of executive functions: a review of our current understanding. Neuropsychol Rev 17:213-233

2. Godefroy O, Martinaud O, Narme P, Joseph PA, Mosca C, Lhommée E, Meulemans T, Czernecki V, Bertola C, Labauge P, Verny M (2018) Dysexecutive disorders and their diagnosis: a position paper. Cortex 109:322-335

3. Chan RC, Shum D, Toulopoulou T, Chen EY (2008) Assessment of executive functions: review of instruments and identification of critical issues. Arch Clin Neuropsychol 23:201-216

4. Moreira HS, Costa AS, Castro SL, Lima CF, Vicente SG (2017) Assessing executive dysfunction in neurodegenerative disorders: a critical review of brief neuropsychological tools. Front Aging Neurosci 9:369

5. Dubois B, Slachevsky A, Litvan I, Pillon BFAB (2000) The FAB: a frontal assessment battery at bedside. Neurology 55:1621-1626

6. Appollonio I, Leone M, Isella V, Piamarta F, Consoli T, Villa ML, Forapani E, Russo A, Nichelli P (2005) The Frontal Assessment Battery (FAB): normative values in an Italian population sample. Neurol Sci 26:108-116

7. Hurtado-Pomares M, Carmen Terol-Cantero M, Sánchez-Pérez A, Peral-Gómez P, Valera-Gran D, Navarrete-Muñoz EM (2018) The frontal assessment battery in clinical practice: a systematic review. Int J Geriatr Psychiatry 33:237-251

8. Iavarone A, Ronga B, Pellegrino L, Loré E, Vitaliano S, Galeone F, Carlomagno S (2004) The Frontal Assessment Battery (FAB): normative data from an Italian sample and performances of patients with Alzheimer's disease and frontotemporal dementia. Funct Neurol 19:191-195

9. Siciliano M, Chiorri C, Battini V, Sant'Elia V, Altieri M, Trojano L, Santangelo G (2019) Regression-based normative data and equivalent scores for Trail Making Test (TMT): an updated Italian normative study. Neurol Sci 40:469-477 
10. Folstein MF, Folstein SE, McHugh PR (1975) "Mini-mental state": a practical method for grading the cognitive state of patients for the clinician. J Psychiatr Res 12:189-198

11. Nasreddine ZS, Phillips NA, Bédirian V, Charbonneau S, Whitehead V, Collin I, Cummings JL, Chertkow H (2005) The Montreal Cognitive Assessment, MoCA: a brief screening tool for mild cognitive impairment. J Am Geriatr Soc 53:695-699

12. Santangelo G, Siciliano M, Pedone R, Vitale C, Falco F, Bisogno R, Siano P, Barone P, Grossi D, Santangelo F, Trojano L (2015) Normative data for the Montreal Cognitive Assessment in an Italian population sample. Neurol Sci 36:585-591

13. Siciliano M, Raimo S, Tufano D, Basile G, Grossi D, Santangelo F, Trojano L, Santangelo G (2016) The Addenbrooke's Cognitive Examination Revised (ACE-R) and its sub-scores: normative values in an Italian population sample. Neurol Sci 37:385-392

14. Conti S, Bonazzi S, Laiacona M, Masina M, Coralli MV (2015) Montreal Cognitive Assessment (MoCA)-Italian version: regression based norms and equivalent scores. Neurol Sci 36:209-214

15. Brugnolo A, De Carli F, Accardo J, Amore M, Bosia LE, Bruzzaniti C, Cappa SF, Cocito L, Colazzo G, Ferrara M, Ghio L (2016) An updated Italian normative dataset for the Stroop color word test (SCWT). Neurol Sci 37:365-372

16. Tremolizzo L, Lizio A, Santangelo G, Diamanti S, Lunetta C, Gerardi F, Messina S, La Foresta S, Riva N, Falzone Y, Filippi M, Woolley SC, Sansone V, Siciliano M, Ferrarese C, Appollonio I, ALS-CBS Italian Study Group (2020) ALS Cognitive Behavioral Screen (ALS-CBS): normative values for the Italian population and clinical usability. Neurol Sci 41:835-841

17. Rothstein HR, Borenstein M, Cohen J, Pollack S (1990) Statistical power analysis for multiple regression/correlation: a computer program. Educ Psychol Measur 50:819-830
18. Champely S (2020) pwr: Basic functions for power analysis (R package version 1.3-0) [Computer software]. The Comprehensive R Archive Network. Available from https://CRAN.R-project.org/ package $=$ pwr. Accessed 20 Feb 2020

19. Kim HY (2013) Statistical notes for clinical researchers: assessing normal distribution (2) using skewness and kurtosis. Restor Dent Endod 38:52-54

20. Capitani E, Laiacona M (2017) Outer and inner tolerance limits: their usefulness for the construction of norms and the standardization of neuropsychological tests. Clin Neuropsychol $31: 1219-1230$

21. Spinnler H, Tognoni G (1987) Standardizzazione e taratura italiana di test neuropsicologici. Ital J Neurol Sci 6:1-120

22. IBM Corp (2021) IBM SPSS Statistics for Windows, Version 27.0. Armonk: IBM Corp

23. R Core Team (2019) R: A Language and environment for statistical computing. (Version 3.6.3) [Computer software]. Retrieved from https://cran.r-project.org. Accessed $20 \mathrm{Feb} 2020$

24. Aiello EN, Depaoli EG (2021) Norms and standardizations in neuropsychology via equivalent scores: software solutions and practical guides. Neurol Sci. https://doi.org/10.1007/ s10072-021-05374-0

25. Siqueira GS, Hagemann PDM, Coelho DDS, Santos FHD, Bertolucci PH (2019) Can MoCA and MMSE be interchangeable cognitive screening tools? A systematic review. Gerontologist 59:e743-e763

Publisher's note Springer Nature remains neutral with regard to jurisdictional claims in published maps and institutional affiliations. 